Check for updates

Cite this: RSC Adv., 2018, 8, 14515

\title{
Dual-sensitive chitosan derivative micelles for site- specific drug release in the treatment of chicken coccidiosis $\uparrow$
}

\author{
Xin Zhang, $\stackrel{+}{a}^{a}$ Gujun Xu,,$^{a}$ Khalid Gadora, ${ }^{a}$ Hao Cheng, ${ }^{a}$ Jin Peng, ${ }^{a}$ Yong Ma, \\ Yang Guo, ${ }^{b}$ Cheng Chi, ${ }^{a}$ Jianping Zhou ${ }^{* a}$ and Yang Ding (DD *a
}

Coccidiosis is a widespread and economic disease that deteriorates the growth of infected animals and largely affects our food safety. It has been proved that chitosan and its derivatives could offer outstanding antibacterial and hemostatic capabilities and help increase weight gain in the breeding of poultry. Herein, we put forward a novel strategy for the development of anti-coccidiosis drug formulations, aiming to synthesize 3-carboxyphenylboronic acid (CPBA) modified chitosan (CS) conjugates which could be self-assembled into polymeric micelles together with diclazuril (DIC), a poorly water-soluble coccidiostat drug, for site-specific drug release in the treatment of animal coccidiosis. The CPBA-modified micelles possessed specific glucose and $\mathrm{pH}$ dual-responsive capacity. The cumulative release of DIC reached the maximum of $85.0 \%$ at $\mathrm{pH} 6.8$ and $50 \mathrm{mM}$ glucose, while only $44.2 \%$ at $\mathrm{pH} 2.0$ and no glucose, which allows drug to be released in the intestinal tract with neutral $\mathrm{pH}$ and glucose existence. ${ }^{1} \mathrm{H}$ NMR and FTIR confirmed the successful synthesis of amphiphilic polymer; and the optimized DIC-loaded CS-CPBA micelles (DIC/CS-CPBA) shared nanoscale particle size of (118.9 \pm 1.1) $\mathrm{nm}$, drug loading of $8.97 \%$ and spherical shape. The pharmacokinetic results indicated that the AUC value of DIC/CS-CPBA micelles was 1.7 times than that of drug suspensions. The anti-coccidial efficacy demonstrated that DIC/CS-CPBA micelles improved the anticoccidial efficacy in vivo and reduced intestinal damage. These results indicated that phenylboronic acid-conjugated chitosan micelles provided a promising platform for specific-targeted drug release in the intestinal tract for the treatment of coccidiosis.

Received 11th March 2018

Accepted 2nd April 2018

DOI: $10.1039 / c 8 r a 02144 a$

rsc.li/rsc-advances gastrointestinal mechanical forces and digestive enzymes, and the released sporozoites invade into the epithelial cells of the intestine for schizogony reproduction to produce merozoites. The resulting merozoites are released into the intestine and undergo gametogony and sporogony reproduction to produce a new generation of oocysts. ${ }^{4}$ Eimeria tenella is considered as one of the most harmful parasite species among genus Eimeria that specifically infects chicken caecum. ${ }^{5}$ Infected chickens usually show clinical symptoms of various degrees, such as bloody diarrhea, retarded weight gain and eventually result in the death of chickens. ${ }^{6}$

Diclazuril (DIC) is a benzeneacetonitrile broad-spectrum coccidiostat which has been widely used for inhibiting the biosynthesis of nucleic acids of E. tenella even at low concentration of $1 \mathrm{ppm}$ in poultry ration against both asexual and sexual reproduction stages of coccidia, but its detailed mechanism is not yet fully understood and needed further studies. ${ }^{7,8}$ It may play a role in the treatment of coccidiosis by improving the immune function in chickens or regulating GAPDH (glyceraldehyde-3-phosphate dehydrogenase) induced apoptosis of the coccidia.,10 However, the extremely low solubility of DIC causes drug sedimentation in aqueous solution, resulting in poor bioavailability. Elevated or
State Key Laboratory of Natural Medicines, Department of Pharmaceutics, Pharmaceutical University, 24 Tongjiaxiang, Nanjing 210009, China. E-mail: zhoujianp60@163.com; dydszyzf@163.com

${ }^{b}$ Institute of Traumatology \& Orthopedics, Nanjing University of Chinese Medicine, Nanjing 210023, China

$\dagger$ Electronic supplementary information (ESI) available. See DOI: 10.1039/c8ra02144a

\$ These authors equally contributed to this work. 
frequent doses of DIC usually lead to drug residues and potential food safety issues. Conventional solubility enhancing techniques, such as solid dispersions ${ }^{11}$ and oral suspensions, ${ }^{12}$ have been introduced to enhance DIC solubility and improve bioavailability and therapeutic outcome. Moreover, due to the particular environment in the gastrointestinal tract of chickens, such as low $\mathrm{pH}$ values and high sugar concentration, ${ }^{13}$ a customized design of drug delivery system is highly needed to allow the drug release at a specific site of the chicken intestine.

Among a variety of polysaccharides, chitosan and its derivatives are the most attractive candidates that have been widely utilized in the pharmaceutical field because of their biocompatibility, biodegradability and biochemical activity. ${ }^{\mathbf{1 4 1 5}}$ The hydroxyl and amino groups on the chitosan make it easy to modify with new functions. For instance, PEGylated chitosan nanoparticles were prepared by Hassani for the delivery of the water-insoluble drug ibuprofen by $\mathrm{O}$-substitution ${ }^{\mathbf{1 6}}$ and Huang modified chitosan by $\gamma$-ray irradiation strategy to achieve the $\mathrm{pH}$ - and thermo-responsive release of drugs. ${ }^{17}$ In addition, the surface mannose partially modified chitosan nanoparticles have been developed for targeting specific types of dendritic cells in the treatment of tumors. ${ }^{18}$ Collectively, the hydrophobic segments grafted onto chitosan would improve the amphiphilic properties of the polymers, which may form self-assembled micelles in water and enable encapsulation of the hydrophobic drugs. ${ }^{19,20}$ Besides, chitosan and its derivatives could enhance the adhesion to the intestinal mucosa and open the intestinal epithelial cells tight junctions (TJs) to promote intestinal absorption. ${ }^{21,22}$ As a food additive for animal feed, chitosan was well known for some benefits such as immunomodulatory, antimicrobial and hypocholesterolemic features to improve the growth rate of broiler chickens. ${ }^{23}$

Boronic acid and its derivatives are a class of non-natural synthetic weak acid, which could be linked to the polyhydroxy group to form a stable covalent complex. ${ }^{24,25}$ In aqueous environment, boronic acid maintains a equilibrium between triangular and tetrahedral forms and both structures can bind to 1,2or 1,3-diols to form a diol-borate complex. Based on this property, boronic acid was conjugated onto graphene oxide (GO) molecularly imprinted polymers (MIPs) as a glycoprotein recognition material, ${ }^{26}$ and further developed as twodimensional (2D) sensors for the detection of cancer biomarkers. ${ }^{27}$ In addition, since many aryl boronic acids do not have mutagenicity and the oral acute toxicity dose of phenylboronic acid is higher than $900 \mathrm{mg} \mathrm{kg}^{-1}$ body weight, it is often used as a safe drug delivery vehicle. ${ }^{28,29}$ Recently, phenylboronic acid (PBA)-based sugar-sensitive materials have drawn the attention in the field of diabetes treatments and related drug delivery system..$^{30,31}$ PBA can explicitly recognize carbohydrate antigens overexpressed on the tumor cell surface after abnormal glycosylation and thus was used for tumor targeted therapy. ${ }^{32}$ Therefore, the polyhydroxy structure presented on the carbohydrate provides an ideal scaffold for these interactions and facilitates the development and utilization of the boric acid based carbohydrate sensors. ${ }^{33,34}$

In this study, the dual-sensitive chitosan-based micelles were proposed after the introduction of the glucose sensitive group, 3-carboxyphenylboronic acid (CPBA), to the backbone of chitosan. The synthesized amphiphilic polymer could self-assemble in water together with drugs to form $\mathrm{pH}$ and glucose dualresponsive micelles. The payload DIC was employed as a model anti-coccidia drug and will be encapsulated into the hydrophobic core of the polymeric micelles. After oral administration, the micellar structure is expected to be stable in the acidic environment of the stomach but release drug rapidly in the chicken's cecum due to the high $\mathrm{pH}$ and glucose concentration for targeted therapy against intestinal parasitic diseases.

\section{Materials and methods}

\subsection{Materials}

Chitosan (molecular weight $=50 \mathrm{kDa}$ and $90 \%$ deacetylated) was purchased from Golden-Shell Pharmaceutical Co., Ltd. (Zhejiang, China). 1-(3-Dimethylaminopropyl)-3ethylcarbodiimide hydrochloride $(\mathrm{EDC} \cdot \mathrm{HCl})$ and $N$-hydroxysuccinimide (NHS) was purchased from Aladdin Reagent Inc. (Shanghai, China). 3-Carboxyphenylboronic acid (CPBA) was obtained from Ark Pharm Inc. (Chicago, USA). Diclazuril was purchased from Zhejiang Guobang Pharmaceutical Co., Ltd (Shaoxing, China) and commercially available suspension of diclazuril were purchased from Jiangxi Bolai Pharmacy Co., Ltd (Jiangxi, China). All the chemical reagents were used as purchased without any further purification.

\subsection{Synthesis of 3-carboxyphenylboronic acid modified chitosan (CS-CPBA)}

The CS-CPBA was synthesized by conjugating the carboxyl groups of CPBA to the amino groups of CS using EDC $\cdot \mathrm{HCl} / \mathrm{NHS}$ amide reaction as one-step procedure depicted in Scheme 1. The procedure was adopted from previous literature with some modification..$^{35}$ Briefly, $100 \mathrm{mg}$ of CS (the primary amino group is about $0.6 \mathrm{mmol}$ ) was dissolved in $20 \mathrm{ml}$ deionized water containing $0.1 \% \mathrm{HCl}$. CPBA was dissolved in $20 \mathrm{ml}$ methanol containing EDC $(0.9 \mathrm{mmol})$ and NHS $(0.9 \mathrm{mmol})$. The reaction was proceeded under stirring for $30 \mathrm{~min}$ at $0{ }^{\circ} \mathrm{C}$ to form active esters of CPBA. The methanol solution containing the active ester of CPBA was added into the chitosan solution, and the reaction mixture was stirred at room temperature for $24 \mathrm{~h}$. The product was dialyzed against distilled water using a dialysis bag (MWCO 12000-14000 Da) for 3 days and then lyophilized to obtain CS-CPBA.

\subsection{Characterization of CS-CPBA}

2.3.1. ${ }^{1} \mathrm{H}$ NMR and FTIR. The chemical structure of CSCPBA dissolved in $\mathrm{D}_{2} \mathrm{O}$ was confirmed by ${ }^{1} \mathrm{H}$ NMR (AMX 600, Bruker, Germany) analysis, and the infrared spectra was tested by Fourier transform infrared spectroscopy (FTIR) (TENSOR 27, Bruker, Germany). The degree of substitution (DS) was defined as the number of CPBA groups conjugated to CS per 100 glucosamine units which could be calculated by the ratio of the proton peak area in ${ }^{1} \mathrm{H}$ NMR.

2.3.2. Critical micelle concentration (CMC). The CMC of amphiphilic CS-CPBA conjugates was evaluated by using 
pyrene as the probe as described in the previous report. ${ }^{36}$ Briefly, $100 \mu \mathrm{l}$ of $\left(6.0 \times 10^{-5}\right) \mathrm{M}$ pyrene dissolved in acetone was added to a series of $10 \mathrm{ml}$ volumetric flasks and then acetone was removed through evaporated under reduced pressure. Different concentrations of CS-CPBA solution $\left(1 \times 10^{-4}\right.$ to $1 \mathrm{mg}$ $\mathrm{ml}^{-1}$ ) were added to the volumetric flask to a final volume of $10 \mathrm{ml}$ and sonicated for $30 \mathrm{~min}$ in water bath. The samples were incubated at $50{ }^{\circ} \mathrm{C}$ for $60 \mathrm{~min}$ and then left to cool down overnight at room temperature. Pyrene fluorescence spectra was obtained by using a fluorescence spectrophotometer (RF-5301 PC, Shimadzu, Japan) with an emission wavelength of $390 \mathrm{~nm}$ and the slit-widths of both excitation and emission was $3 \mathrm{~nm}$. The CMC value was estimated by the intersection point when extrapolating the intensity ratio $I_{338} / I_{333}$ of excitation wavelength at low and high concentration regions.

\subsection{Preparation and characterization of DIC/CS-CPBA micelles}

2.4.1. Preparation of DIC/CS-CPBA micelles. The diclazurilloaded polymeric micelles (DIC/CS-CPBA) were prepared with dialysis method. Briefly, $20 \mathrm{mg}$ of CS-CPBA polymers were dispersed in $3 \mathrm{ml}$ of distilled water until the product completely dissolved, $3 \mathrm{mg}$ of DIC was dissolved in $300 \mu \mathrm{l}$ of $\mathrm{N}, \mathrm{N}$-dimethylformamide (DMF) and added drop wise to the CS-CPBA solution while stirring. The mixture was stirred for another $10 \mathrm{~min}$ at $25^{\circ} \mathrm{C}$ followed by sonication for $20 \mathrm{~min}$ at $100 \mathrm{~W}$ using a probe-type sonicator (Beidi-IIYJ, Nanjing Beidi equipment Co., Ltd, China) in an ice-water bath. Then the resulted products were dialyzed against distilled water in a dialysis bag (MWCO 12000$14000 \mathrm{Da}$ ) for $24 \mathrm{~h}$. The supernatant was collected by centrifugation at $8000 \mathrm{rpm}$ for $10 \mathrm{~min}$ and filtered through a $0.8 \mu \mathrm{m}$ syringe filter to remove the unloaded DIC.

2.4.2. Drug loading content and entrapment efficiency. The amount of DIC entrapped in CS-CPBA micelles was measured by UV absorbance at $280 \mathrm{~nm}$ using high performance liquid chromatography (HPLC, LC-2010, Shimadzu, Japan). The mobile phase was a 57/43 (v/v) mixture of acetonitrile/water with a flow rate of $1.0 \mathrm{ml} \mathrm{min}{ }^{-1}$. The following formula was used to calculate the drug-loading (DL\%, formula (1)) and entrapment efficiency (EE\%, formula (2)), and the values were reported as mean \pm standard deviation (SD) $(n=3)$.

$$
\mathrm{DL}(\%)=\frac{\text { mass of diclazuril encapsulate in micelles }}{\text { total mass of micelles }} \times 100 \%
$$

$$
\begin{aligned}
\mathrm{EE}(\%)= & \frac{\text { mass of diclazuril encapsulate in micelles }}{\text { mass of diclazuril added to micelle preparation }} \\
& \times 100 \%
\end{aligned}
$$

2.4.3. Particle size and polydispersity index (PDI). The particle size and PDI of DIC/CS-CPBA micelles were determined in an aqueous dispersion at $5 \mathrm{mg} \mathrm{ml}^{-1}$ at $25{ }^{\circ} \mathrm{C}$ using a Zetasizer (3000HS, Malvern Instruments Ltd, UK). The PDI values were assessed by determination of particle electrophoretic velocity using the same instrument. To determine the storage stability of
DIC/CS-CPBA micelles, the samples were stored at $25{ }^{\circ} \mathrm{C}$ without direct light. The appearance, average particle size and PDI were observed to evaluate the stability of micelles for 7 days after treatment. $^{37}$

2.4.4. Morphology and glucose sensitivity. To determine the change of particle size in the presence of glucose, CS-CPBA micelles containing DIC were prepared according to the aforementioned method. The DIC/CS-CPBA micelles were incubated with $20 \mathrm{mM}$ glucose solution at $\mathrm{pH}$ 6.8, and the changes in the micelle size and PDI were determined by using the dynamic light scattering (DLS) method. The morphology of DIC/CS-CPBA micelles were visualized under transmission electron microscopy (TEM, H-7000, Hitachi, Japan) and atomic force microscopy (AFM, SPA 3800N, Seiko, Japan) under conditions of no glucose (pure water) or $20 \mathrm{mM}$ glucose ( $\mathrm{pH}$ 6.8). The negatively stained samples for TEM analysis were air-dried and performed upon 100 $\mathrm{kV}$ of the accelerating voltage. Meanwhile, AFM was proceeded in tapping mode and the widths of the particles were measured using Nanoscope version 7.3 software. Furthermore, size changes of DIC/CS-CPBA micelles at glucose concentrations of 20, 50, $100 \mathrm{mM}$ was measured with DLS. Briefly, the drug-loaded micelles were incubated with a different concentration of glucose solution at $37^{\circ} \mathrm{C}$ in an orbital shaker. The particle size of the micellar solution was measured at predetermined time intervals of $0,5,15,30 \mathrm{~min}$, and 1, 2, 3, 4, 5, 6, 12, $24 \mathrm{~h}$.

2.4.5. X-ray diffraction (XRD) and differential scanning calorimetric (DSC) analysis. To determine the solid state of encapsulated drug, X-ray diffraction spectroscopy (XRD) and differential scanning calorimetric (DSC) analysis were performed on the physical mixture of DIC and CS-CPBA and DIC/ CS-CPBA micelles, respectively. XRD analysis was accomplished by a powder diffraction meter (XD-3A, Bruker, Germany) with $\mathrm{Cu} \mathrm{K-alpha} \mathrm{radiation.} \mathrm{Samples} \mathrm{were} \mathrm{scanned} \mathrm{from} 5$ to $40^{\circ}$ at scanning speed of $1^{\circ} \min ^{-1}$ and a step size of $0.05^{\circ}$. DSC analysis was executed using an equipment (DSC 204, NETZSCH, Germany) with the temperature and heating rate of $40-300{ }^{\circ} \mathrm{C}$ and $10{ }^{\circ} \mathrm{C} \min ^{-1}$, respectively.

\subsection{Glucose and pH-triggered drug release}

The in vitro drug release profiles from DIC/CS-CPBA micelles were studied by dialyzing the drug-loaded micellar preparations against $200 \mathrm{ml}$ of PBS buffer $(0.01 \mathrm{M})$ containing $0.5 \%(\mathrm{v} / \mathrm{v})$ Tween-80 with different glucose concentration (20 or $50 \mathrm{mM})$. The $\mathrm{pH}$ values of $2.0,5.5$ and 6.8 were used to simulate $\mathrm{pH}$ environment of the chicken stomach, small intestine and cecum, respectively; while different concentrations of glucose were used to simulate postprandial glucose concentrations in the gastro-intestine. In predetermined time intervals, $2 \mathrm{ml}$ of released medium was withdrawn and replenished with an equal volume of fresh medium. The amount of released DIC was detected by HPLC method with UV detection at $280 \mathrm{~nm}$, and the mobile phase was a $57 / 43(\mathrm{v} / \mathrm{v})$ mixture of the acetonitrile/0.2\% aqueous phosphoric acid solution with a flow rate of 1.0 $\mathrm{ml} \mathrm{min}{ }^{-1}$. The concentration of DIC in the samples was determined by measuring peak areas of HPLC, and the cumulative release was calculated and then plotted against time. 


\subsection{In vitro cytotoxicity}

The in vitro cytotoxicities of blank CS-CPBA carrier, free DIC, DIC/CS-CPBA micelles, and the mixture of DIC and CS-CPBA conjugates were determined by measuring cell viability on human colon cancer cell line Caco-2 using MTT assay. Caco-2 cells were cultured in 96-well plates at $5 \times 10^{3}$ cells per well for $24 \mathrm{~h}$ at $37{ }^{\circ} \mathrm{C}$ and $5 \% \mathrm{CO}_{2}$ in Dulbecco's modified Eagle's medium (DMEM) containing 10\% fetal bovine serum. The cells were then treated with free DIC, DIC/CS-CPBA micelles and the mixture of DIC and CS-CPBA conjugates, which were dissolved in DMEM medium containing different drug concentrations of $0.001,0.01,0.1,1,10$ and $100 \mu \mathrm{g} \mathrm{ml}^{-1}$. The concentration of blank CS-CPBA carrier was set at equivalent concentration of DIC/CS-CPBA micelles. Thereafter, $20 \mu \mathrm{l}$ of MTT $\left(5 \mathrm{mg} \mathrm{ml}^{-1}\right)$ was added to each wells and the plates were incubated for another $4 \mathrm{~h}$ at $37{ }^{\circ} \mathrm{C}$. The formed formazan crystals were subsequently dissolved by $100 \mu \mathrm{l}$ of dimethyl sulfoxide (DMSO). The absorbance was recorded at $570 \mathrm{~nm}$ measured by a microplate reader (EL800, BIO-TEK Instruments Inc., USA). Untreated cells were used as a control group and the cell viability (\%) was calculated by the following formula (3):

$$
\text { Cell viability }(\%)=A_{\text {test }} / A_{\text {control }} \times 100 \% \text {. }
$$

\subsection{In vivo anti-coccidial efficacy}

2.7.1. Birds and management. All animal experiments were conducted under a protocol approved by China Pharmaceutical University Ethics Committee and in accordance with the National Institutes of Health guide for the care and use of Laboratory animals (NIH publications no. 8523, revised 1985). A total of 100 male Wen's broiler chickens aged 1 day were purchased from a local hatchery (Nanjing, China) and kept on adaptive feeding for 14 days. At the 15th day of age, 40 healthy chickens with similar weight and no oocysts existence verified by fecal examination were randomly divided into 4 groups, including uninfected control group (without coccidia challenge), infected control group (with coccidia challenge), DIC/ CS-CPBA micelles (coccidia challenged and supplemented with drug-loaded micelles) and DIC suspension group (coccidia challenged and supplemented with commercially available DIC suspension), and housed in pens of identical size with a single tray per group to catch fecal material.

2.7.2. Induction of coccidia infection. The chickens are kept in a coccidia-free environment where each group of diets and drinking utensils is treated separately to prevent crossinfection. Coccidia oocysts were provided by Nanjing Agricultural University and isolated from cecum contents of chickens infected with Eimeria tenella as described by previous articles. ${ }^{38}$ The coccidia oocysts were stored in potassium dichromate solution, which is diluted with distilled water to a concentration of $1.0 \times 10^{5}$ spores per ml. Groups of infection, DIC/CS-CPBA micelles, and DIC were inoculated with $1 \mathrm{ml}$ of coccidiosis oocysts solution by oral administration, and the uninfected group was inoculated with the same amount of distilled water.
2.7.3. Anti-coccidial efficacy. Anti-coccidial efficacy of treatments was evaluated by bloody diarrhea, intestinal lesion score, body weight gain (BWG) and oocyst counts. Bloody diarrhea, which reflects the severity of coccidiosis, was monitored during the period of the 5th to the 7th day after challenge. The intestinal lesion score of each group was calculated according to the reported method, and divided into 0 to 4 points based on the epithelial color, fluid accumulation and the overall general appearance of the intestine $(0=$ no lesion, $1=$ mild lesion, $2=$ moderate lesion, $3=$ severe lesion, $4=$ more severe lesion). ${ }^{39}$

The body weight of each chicken was recorded daily during the experiment and BWGs were obtained by subtracting the initial average weight from the final average weight. The feed intake of each group was obtained by measuring the feed consumption during the experiment. Feed conversion ratio (FCR) was calculated by the following formula (4):

$$
\mathrm{FCR}=\text { feed intake/weight gain } .
$$

The peak excretion of oocysts was recorded on the 7th day after infection, and oocysts of pooled feces were counted by McMaster's method at the 5th, 6 th and 7 th day after challenge. ${ }^{\mathbf{4 0}}$ The average number of oocysts in three days expressed as oocyst output per gram (OPG). On necropsy of birds, cecum tissue samples were collected and fixed in $10 \%$ buffer paraformaldehyde. The tissue sections were stained and then examined under a microscopic to observe the lesions like hemorrhage, coccidian oocysts infection, and villi erosion.

\subsection{In vivo pharmacokinetics assessment}

2.8.1. Birds and management. Twelve healthy male Wen's broiler chickens with an average body weight of $1.5 \mathrm{~kg}$ and 45 days old age were used. Chickens were fed on a balanced portion free from any anticoccidial and antibiotic drugs for 7 days before the experiment to ensure that animals without anticoccidia drug residues.

2.8.2. Experimental design. Wen's broiler chickens divided into two groups $(n=6)$ : DIC/CS-CPBA group (chickens treated with drug-loaded CS-CPBA micelles at the single dose of $15 \mathrm{mg}$ $\mathrm{kg}^{-1}$ ) and DIC suspension group (chickens treated with DIC suspension containing $0.5 \%$ CMC-Na in accordance with the single dose of $15 \mathrm{mg} \mathrm{kg}^{-1}$ ). Blood samples were taken from the wing vein of each chicken at certain time points $(0,15,30 \mathrm{~min}$ and 1, 2, 3, 4, 8, 12, 24, 36, $48 \mathrm{~h}$ ) post oral administration. The samples were centrifuged at $4000 \mathrm{rpm}$ for $10 \mathrm{~min}$ and the supernatant plasma was collected, then stored at $-20{ }^{\circ} \mathrm{C}$ until further use.

2.8.3. Analytical method. The amount of DIC in the plasma was determined using HPLC with UV detection at $280 \mathrm{~nm}$. The mobile phase was a 55/25/20 (v/v/v) mixture of acetonitrile/ water $/ 0.1 \%$ trifluoroacetic acid with a flow rate of $1.0 \mathrm{ml} \mathrm{min}{ }^{-1}$. A standard curve for DIC was constructed by using antibioticfree pooled plasma samples collected from two nonmedicated chickens treated by the same procedure. Pharmacokinetic parameters of DIC were calculated by DRUG AND 
STATISTICS software (DAS, version 2.1.1, Mathematical Pharmacology Professional Committee of China).

\subsection{Statistical analysis}

Statistical analysis was performed using SPSS 23.0 statistical software (SPSS Inc., USA). The obtained results represented as mean \pm S.D. Statistical significance between two groups were analyzed by Student's $t$-test. Differences significance was established at $* p<0.05,{ }^{* *} p<0.01$, and ${ }^{* * *} p<0.001$.

\section{Results and discussion}

\subsection{Synthesis and characterization of CS-CPBA conjugates}

The synthetic procedure of CS-CPBA conjugates in Scheme 1 showed that the chitosan (CS) provided free amino groups that could be conveniently conjugated with the free carboxylic acid groups on 3-carboxyphenylboronic acid (CPBA) by amide reaction. The ${ }^{1} \mathrm{H}$ NMR spectrum of the raw material CS and the synthetic product CS-CPBA pointed the CPBA peaks at $\delta=7.4-$ $8.2 \mathrm{ppm}$ and the proton peaks at $\delta=3.0-4.0 \mathrm{ppm}$ belonged to $\mathrm{C}_{3}-\mathrm{C}_{6}$ of chitosan unit (Fig. 1A). Moreover, the proton peaks with chemical shift $\delta=2.9 \mathrm{ppm}$ belonged to the methylene group of CS, and the proton peak at $\delta=2.0 \mathrm{ppm}$ related to the methyl group which was from partially acetylated CS. ${ }^{41}$ The above characteristic peaks indicated that the activated group of CPBA has been successfully grafted onto the chitosan backbone. The Fourier transform infrared spectra (FTIR) of CS and CS-CPBA demonstrated that there was a significant blunt peak at around $3400 \mathrm{~cm}^{-1}$, which was related to the hydrogen bonds of free hydroxyl groups on chitosan (Fig. 1B). Compared to the infrared spectrum of CS, the absorption band at $1714.2 \mathrm{~cm}^{-1}$ of CS-CPBA conjugates is the characteristic peak of the carbonyl group, demonstrating the presence of the - $\mathrm{CONH}-$ group. The range of $900-650 \mathrm{~cm}^{-1}$ is the absorption band of the benzene ring, and the sharp peaks appearing at $708.7 \mathrm{~cm}^{-1}$ also indicated the presence of di-substituted benzene. The degree of substitution was estimated about $23 \%$ for the feed molar ratios

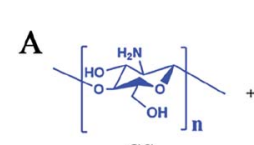

CS

B

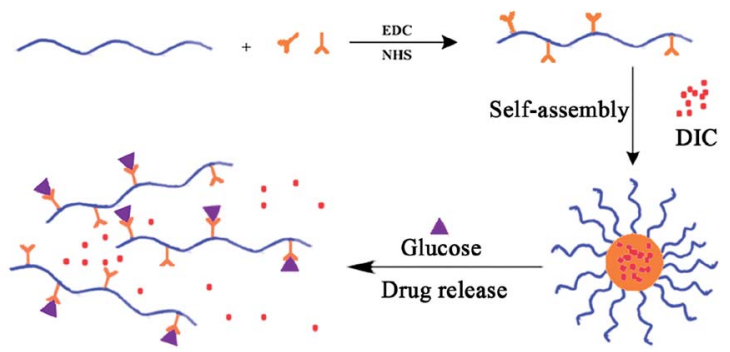

Scheme 1 (A) The synthesis of chitosan-CPBA (CS-CPBA). (B) The formation of drug-loaded micelles, the micellar structure dissemble, and drug release in the presence of glucose. of $1.5: 1$, by calculating the relative peak areas between the original acetamide group of CS at $\delta=2.0$ and CPBA group at $\delta=$ $7.4-8.2 .{ }^{42}$

The critical micellar concentration (CMC) value means the lowest concentration of conjugates that can be self-assembled to form the amphiphilic micelles, which was determined by using a fluorescence probe to assess the stability of amphiphilic micelles. ${ }^{43}$ As reported, the fluorescence intensity ratio of $I_{338} /$ $I_{333}$ is plotted against the log of polymer concentration. ${ }^{44}$ The intersection of the two tangential lines indicated the successful formation of the polymer micelles and the CMC value of CSCPBA conjugates was nearly $17.78 \mu \mathrm{g} \mathrm{ml}^{-1}$ (ESI Fig. S1†). This relatively low CMC value implied that CS-CPBA micelles would remain stable even after dilution in vivo; ${ }^{45}$ and it is therefore beneficial for improving the drug delivery efficiency in blood circulation.

\subsection{Preparation and characterization of DIC/CS-CPBA micelles}

Characterizations including drug loading (DL), encapsulation efficiency (EE), particle size and polydispersity index (PDI) of DIC/CS-CPBA micelles were collected and demonstrated in Table 1. It showed that the particle size of the drug-loaded micelles revealed significant changes with the increase of the feed ratio of CPBA, and the minimum value of particle size was $(118.9 \pm 1.1) \mathrm{nm}$ at the ratio of $1.5: 1$ (CPBA : CS). At the ratio of $2: 1$ (CPBA : CS), the particle size of DIC/CS-CPBA micelles exceeded $(218.5 \pm 2.6) \mathrm{nm}$, indicating that the increase of the hydrophobic CPBA groups may affect the particle size of micelles. Moreover, DL\% and EE\% of DIC/CS-CPBA micelles were increased with the addition of CPBA. At the feed ratio of 1.5 : 1 (CPBA : CS), the synthesized product could form stable micellar structure together with DIC incorporation, and the drug loading efficiency reached the maximum of $8.97 \%$ and PDI of 0.061 , which also indicated a narrow particle size distribution. Accordingly, the feed ratio of 1.5:1 (CPBA:CS) was applied as the optimal ratio for the subsequent experiments. In addition, the amount of DIC embedded into DIC/CS-CPBA micelles was up to $0.6 \mathrm{mg} \mathrm{ml}^{-1}$, which represented nearly 1000 times of drug solubility compared to that of DIC in water at $0.69 \mu \mathrm{g} \mathrm{ml}{ }^{-1} .^{11}$

\subsection{Morphological observation and particle size variation}

The particle size of the obtained DIC/CS-CPBA micelles was determined as $(118.9 \pm 1.1) \mathrm{nm}$ with a narrow PDI value of $(0.061 \pm 0.033)$. After adding a $20 \mathrm{mM}$ glucose solution ( $\mathrm{pH} 6.8)$, the particle size increased to $(178.0 \pm 3.1) \mathrm{nm}$, and the PDI of the micelles also increased to $(0.286 \pm 0.015)$. As shown in Fig. 2A, it can be seen that a single diameter peak transferred into double particle size distribution in the size range of 50$500 \mathrm{~nm}$, suggesting that the micellar nanoparticles may partially dissociate and become loose upon the glucose response, resulting in variations of average particle size and PDI value. TEM images demonstrated that DIC/CS-CPBA micelles shared spherical and compact morphological feature (Fig. 2B), and the particle size was approximately $100 \mathrm{~nm}$ that was smaller 


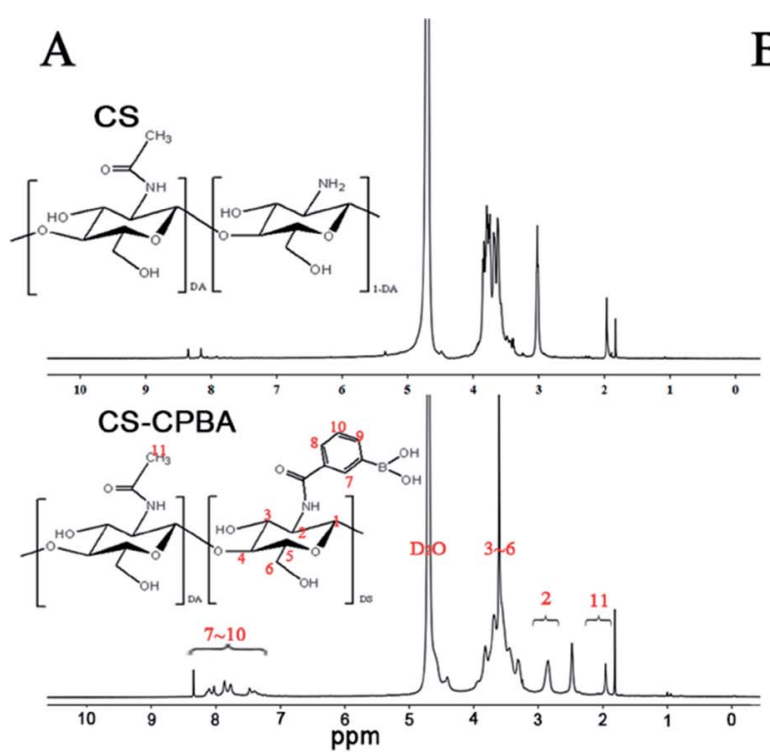

B

Fig. $1{ }^{1} \mathrm{H}$ NMR (A) and FTIR (B) spectra of CS, CS-CPBA copolymer.

than the hydrodynamic diameter obtained from DLS experiment. This may be due to the collapse of the surface hydration layer of the particles during drying processes. ${ }^{46,47}$ However, when dissolved in a $20 \mathrm{mM}$ glucose solution ( $\mathrm{pH}$ 6.8), the particle size of DIC/CS-CPBA micelles increased, and the spherical structure of the micelles became cracked and incomplete as indicated by the arrow. As shown in Fig. 2C, AFM images revealed the similar morphological transformation, and the three-dimensional spectrum of the DIC/CS-CPBA micelles suggested that the spherical nanoparticles are enlarged in the high glucose concentration and $\mathrm{pH}$ value, indicating the fusion of collapsed micelles. The increase of particle size may be ascribed to the decrease of chitosan solubility at high $\mathrm{pH}$ value, ${ }^{48}$ and CPBA induced destruction of micellar hydrophilicity and hydrophobicity balance under glucose-trigger. As shown in Fig. 2D, the particle size of the micellar solution absent of glucose was substantially constant; while the presence of glucose resulted in a parallel increase in particle size. The higher glucose concentration, the larger particle size of micellar nanoparticles. The CPBA groups in nanostructure gave a glucose responsiveness that was capable of binding to 1,2diols or 1,3-diols structure of glucose, resulting in enhanced hydrophilicity of the polymer and a gradual increase in particle size. Since the glucose concentration in the intestine after animal feeding could reach a high level of $50 \mathrm{mM},{ }^{49,50}$ consequently the DIC/CS-CPBA micelles would swell and cause the particle size to increase, which is beneficial for site-specific drug release in intestinal lesion. The storage stability of the drugloaded micelles for 7 days was further evaluated. As demonstrated in Fig. 2E, there was slight variation in particle size and PDI of DIC/CS-CPBA micelles, and the particle size was in the range of 118.9-110.9 $\mathrm{nm}$. The stability of DIC/CS-CPBA micelles indicated low aggregation probability, mainly due to the spatial effect of the hydrated layer formed on the particle surface and the repulsive force between the nanoparticles. ${ }^{51}$

\subsection{XRD and DSC analysis}

To confirm drug crystalline state, samples including DIC, physical mixture of DIC and CS-CPBA, and DIC/CS-CPBA micelles have been investigated by using XRD and DSC analyses, respectively. In Fig. 3A, DIC/CS-CPBA micelles and physical mixture shared the similar broad hump in the lower $2 \theta$ region of $0^{\circ}$ to $11^{\circ}$, which may indicate that the presence of an amorphous construction in the samples or the background of the glass substrate. ${ }^{52}$ Moreover, when DIC physically mixed with CS-CPBA conjugates, the characteristic crystal peaks related to DIC at $2 \theta$ of $15.5^{\circ}$ was still observed; while there was no DIC peaks in the lyophilized DIC/CS-CPBA group. Thus, it was

Table 1 Drug loading (DL), encapsulation efficiency (EE), particle size and polydispersity index of DIC/CS-CPBA. Data presented as mean \pm S.D., $n=3$

\begin{tabular}{|c|c|c|c|c|}
\hline $\begin{array}{l}\text { The feed ratio } \\
\text { of CPBA/CS unit }\left(\mathrm{mol} \mathrm{mol}^{-1}\right)\end{array}$ & DL (\%) & EE $(\%)$ & Particle size (nm) & Polydispersity index \\
\hline $0.5: 1$ & $3.43 \pm 0.47$ & $27.47 \pm 2.65$ & $139.5 \pm 1.0$ & $0.144 \pm 0.091$ \\
\hline $1.5: 1$ & $8.97 \pm 1.59$ & $59.13 \pm 4.29$ & $118.9 \pm 1.1$ & $0.061 \pm 0.033$ \\
\hline $2.0: 1$ & $8.20 \pm 1.40$ & $61.28 \pm 5.78$ & $218.5 \pm 2.6$ & $0.266 \pm 0.017$ \\
\hline
\end{tabular}




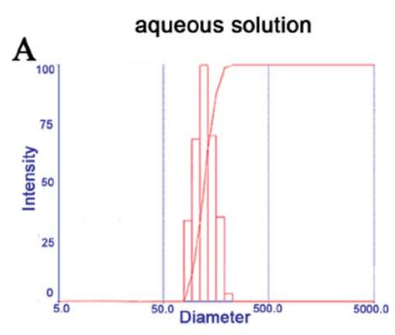

B

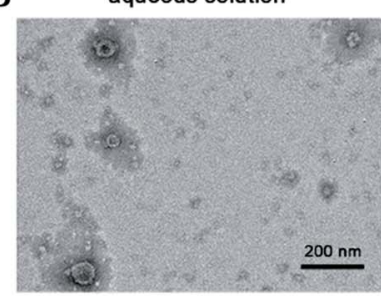

pH $6.8+20 \mathrm{mM}$ glucose

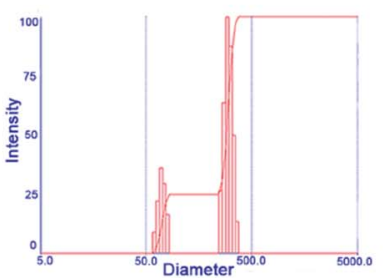

$\mathrm{pH} 6.8+20 \mathrm{mM}$ glucose

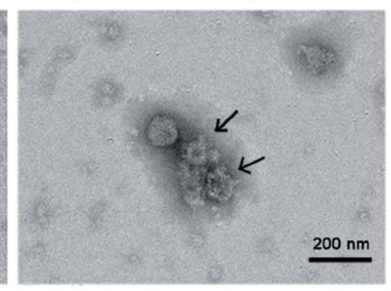

C

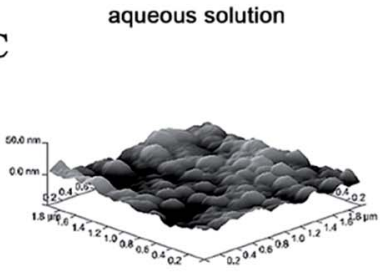

$\mathrm{pH} 6.8+20 \mathrm{mM}$ glucose

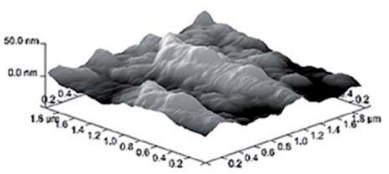

$\mathrm{E}$

D

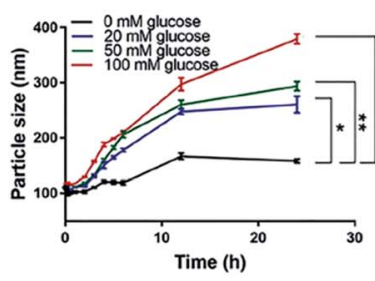

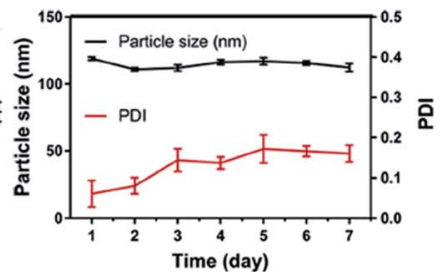

Fig. 2 (A) The changes of particle size in aqueous solution and $20 \mathrm{mM}$ glucose solution (pH 6.8). TEM (B) and AFM (C) images of the DIC/CSCPBA micelles incubated in different media, including aqueous solution and $20 \mathrm{mM}$ glucose solution (pH 6.8). (D) The changes of micellar particle size in different concentrations $(0,20,50$ and $100 \mathrm{mM}$ ) of glucose. (E) The particle size and polydispersity index (PDI) changes for 7 days storage.

concluded that DIC molecules were amorphous in micelles. As DSC diagrams shown in Fig. 3B, the endothermic peak at $296^{\circ} \mathrm{C}$ in DSC thermogram of DIC group was attributed to the decomposition of DIC; however, the endothermic peak of DIC did not exist in the thermogram of DIC/CS-CPBA micelles but in those of physical mixture of CS-CPBA and DIC, which also suggested that DIC encapsulated into the micelles was amorphous. $^{53}$

\subsection{Glucose and pH-triggered drug release}

The drug release profile was investigated at $37{ }^{\circ} \mathrm{C}$ in PBS at different $\mathrm{pH}$ values and glucose concentrations. As demonstrated in Fig. 4A, typical two-phase-release profiles were observed as sustained release and rapid release phase. The rapid release phase will inhibit the growth of the coccidia by increasing the concentration of drug in short time, while the sustained release will provide the possibility of a sustained anticoccidia effect. Moreover, when there is no glucose, the cumulative release of DIC was increased as the value of $\mathrm{pH}$ increased. At $\mathrm{pH} 2.0$ of chicken stomach environment, the cumulative release of DIC reached $44.2 \%$, less than that at pH 5.5 (46.6\%) and $6.8(55.7 \%)$ after $72 \mathrm{~h}$. For explanation, the free amino groups on chitosan in DIC/CS-CPBA micelles remain protonated in aqueous solution, thus restricted the swelling and drug release at low $\mathrm{pH}$. However, at high $\mathrm{pH}$, the amino groups deprotonate and alter the ionic interaction between the polymer chains..$^{54}$ The $\mathrm{pH}$-dependent release profile suggested that DIC/ CS-CPBA micelles were more likely to release the drug in the chicken intestine at $\mathrm{pH}$ 6.8. ${ }^{55,56}$ To further demonstrate that

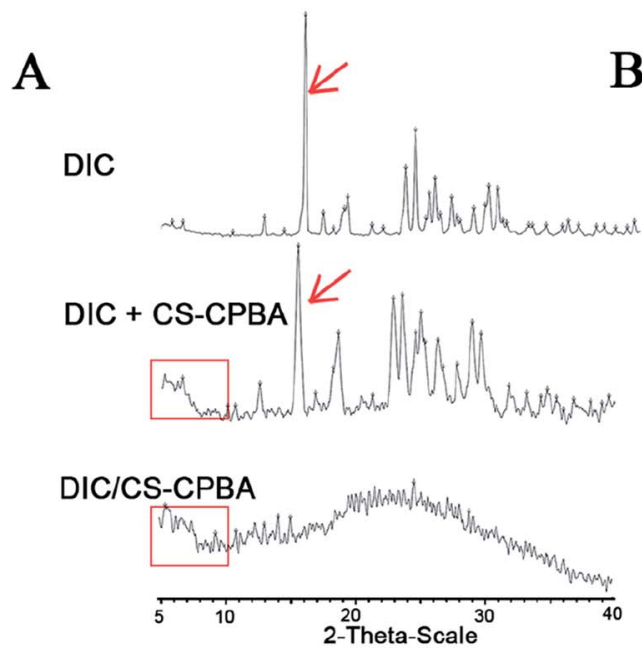

B
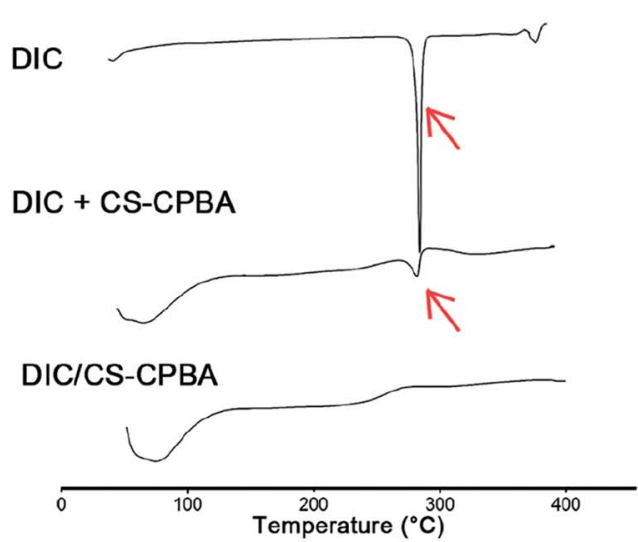

Fig. 3 XRD (A) and DSC (B) analysis of DIC, physical mixture of DIC and CS-CPBA, and DIC/CS-CPBA micelles. 
A

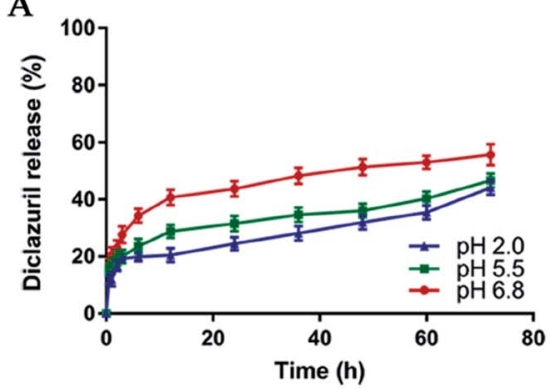

B

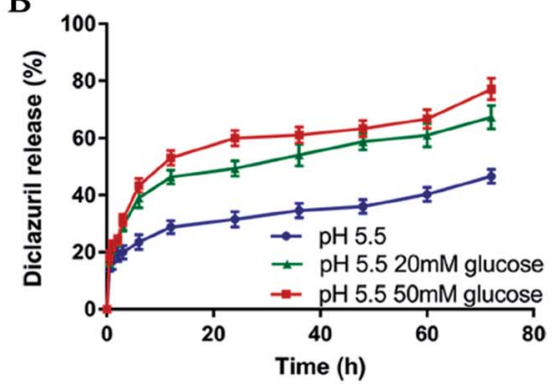

C

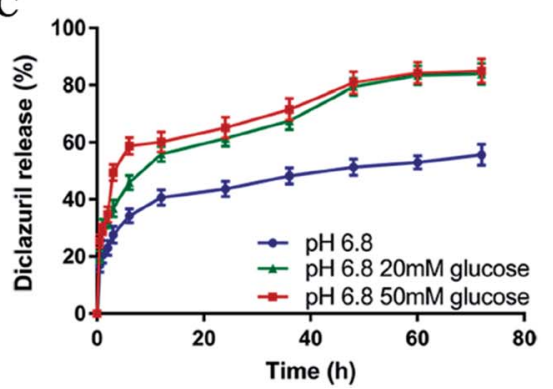

Fig. 4 (A) Drug release profile of DIC/CS-CPBA micelles at different $\mathrm{pH}$ conditions, including $\mathrm{pH} 2.0, \mathrm{pH} 5.5$ and $\mathrm{pH} 6.8$. Drug release profile of DIC/CS-CPBA micelles under glucose concentrations of $20 \mathrm{mM}$ and $50 \mathrm{mM}$ at pH 5.5 (B) and pH 6.8 (C).

DIC/CS-CPBA micelles could manage a glucose-responsive drug controlled release, we introduced different glucose concentrations of 20 or $50 \mathrm{mM}$ for the stimulation of gut environment (Fig. 4B and C). The cumulative release of DIC was dramatically increased in the presence of glucose $(50 \mathrm{mM})$ and reached the maximum of $85.0 \%$ at $\mathrm{pH}$ 6.8. Structurally, the negatively charged CPBA can combine with the polyhydroxy groups of glucose for the formation of stable and soluble phenyl borates. The glucose-triggered drug release mediated by CPBA-based micelles has been widely demonstrated in the gastrointestinal tract for insulin and other drugs, and the degree of cumulative release is related to the concentration of glucose. Similarly, upon high $\mathrm{pH}$ value and glucose concentration in the animal intestine, DIC/CS-CPBA micelles could mediate site-specific drug release for the treatment of intestinal parasitic diseases in animals.

\subsection{In vitro cytotoxicity assay}

The cytotoxicity of DIC/CS-CPBA micelles against human colon cancer cell line Caco-2 was examined by MTT assay. DIC had low acute toxicity and was not mutagenic, genotoxic, carcinogenic, embryotoxic, fetotoxic or teratogenic. ${ }^{57,58}$ The cell viability of Caco-2 cells gradually decreased with the increase of free drug concentration. However, the cytotoxicity results of CS-CPBA polymers (Fig. 5A) and DIC/CS-CPBA micelles (Fig. 5B) indicated that the drug-free carriers and drug-loaded formulations were less toxic than the free drug and there was no essential cell death at the DIC concentration of $1 \mu \mathrm{g} \mathrm{ml}{ }^{-1}$. The cytotoxicity of the physical mixture of drug and carrier was lower than that of the free drug, which may be due to the self-assembly of drugloaded micelles in the medium. The newly assembled micelles incorporated a portion of free drugs and reduced cell cytotoxicity. Respectively, the cell viability of DIC/CS-CPBA micelles was significantly higher than that of free drug, possibly due to the decreased drug toxicity of micellar structure. The results of the cell assay indicated that DIC/CS-CPBA micelles were effective at lower cytotoxicity of drugs for oral administration to reduce intestinal cell damage.

\subsection{In vivo anti-coccidia efficacy}

The anti-coccidia activities were assessed in infected broiler chicks to verify the possible applications of DIC/CS-CPBA micelles for coccidiosis treatment. As seen in ESI Table S1, $\dagger$ there was no bloody diarrhea in the uninfected chicks. For comparision, bloody diarrhea in DIC/CS-CPBA treatment group was significantly less than that in the infected and DIC suspension treated group, indicating that the animals treated by DIC/CS-CPBA micelles gave the mildest coccidia infection and the outstanding therapeutic efficacy. The body weight gain (BWG) of the chicks were presented in Table 2. By comparing the average weight changes of each group before and after the experiment, we found that the BWG of the uninfected control group was the largest; while, DIC/CS-CPBA treated group demonstrated the highest relative BWG among the infected chickens. Moreover, the value of FCR indicated the feed utilization ability of chicks, the smaller value of FCR, the higher feed
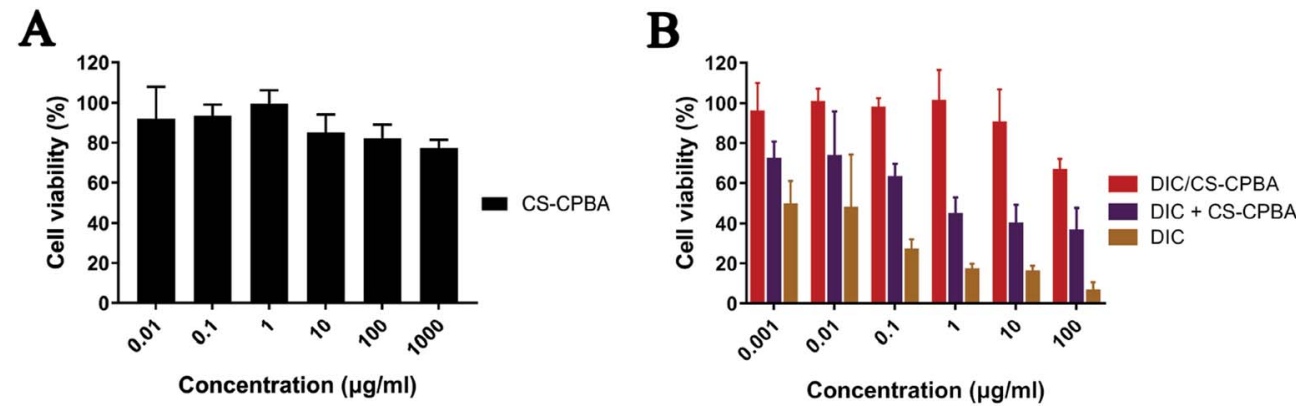

Fig. 5 Cell viability of Caco-2 cells after incubation of CS-CPBA polymers (A) and DIC formulations (B) for $24 \mathrm{~h}$ with different concentrations. Data presented as mean \pm S.D. $(n=5)$. 
Table 2 Average body weight, relative body weight gain (\%) and feed conversion ratio (FCR) determined at the initial and the end of the experiment. Data presented as mean \pm S.D., $n=10$

\begin{tabular}{lllll}
\hline & \multicolumn{2}{l}{ Average body weight $(\mathrm{g})$} & & \\
\cline { 2 - 3 } Groups & Before challenge & After challenge & Relative body weight gain (\%) \\
\hline Uninfected control & $95.8 \pm 5.7$ & $173.3 \pm 13.8$ & FCR \\
Infected control & $96.4 \pm 4.8$ & $138.2 \pm 14.3$ & 53 & 3.06 \\
DIC/CS-CPBA & $96.4 \pm 7.2$ & $153.9 \pm 21.6$ & 5.42 \\
DIC suspensions & $96.3 \pm 5.8$ & $141.0 \pm 17.9$ & 57 & 3.88 \\
& & & &
\end{tabular}

utilization ability and the better health of the chickens. As shown in Table 2, the feed utilization ability among groups decreased in the order of the uninfected > DIC/CS-CPBA micelles $>$ DIC suspensions $>$ the infected. The results revealed that DIC/CS-CPBA micelles were not only conducive to performing the practical prevention and treatment of coccidiosis, but also increasing body weight and reducing feed consumption. The results of weight gain truly reflected the inherent efficacy of the synthesized chitosan carrier in the growth improvement of broiler chicks. The results of the intestinal lesions score were presented in ESI Table S2, $\dagger$ and DIC/CSCPBA micelles treated group was provided with a smallest score of $(0.12 \pm 0.35)$ compared with other coccidiosis groups. Low lesion scores inferred that the intestine suffered from less bleeding symptoms, demonstrating that DIC carried by CSCPBA micelles inhibited the proliferation of coccidia, and the available positive charge of $\mathrm{NH}^{3+}$ residues on chitosan polymer chain might lead to the antibacterial effect of the formulation and weaken intestinal damage. ${ }^{59,60}$

Body weight variations of chicks in different groups were presented in Fig. 6A. Chicks without coccidia infection possessed a faster weight gain than DIC/CS-CPBA micelles treated group; and no significant differences in body weight changes between groups of DIC suspensions and the infected control group. Furthermore, the feces of the experimental chicks were collected on the $5^{\text {th }}, 6^{\text {th }}$ and $7^{\text {th }}$ day after the infection. According to Fig. 6B, the number of oocysts per gram of feces (OPG) was counted; and no coccidia oocysts were detected in the fecal contents of the uninfected chickens but many oocysts were found in the cecum of the infected chickens. The number of oocysts in DIC/CS-CPBA micelles treatment group was less than other infected groups. To study the pathological features, Fig. 6C presented the cecum images obtained from chicks after dissection at $8^{\text {th }}$ day. Compared to the uninfected and DIC/CS-CPBA micelles treated groups, the cecum walls of the infected and DIC suspension treated chicks were thicker, and the cecum contents were darker and more bleeding occurrence was happened. The outstanding performance of anticoccidiosis treated with micellar formulations may be attributed to the combination effects of chitosan and drug DIC. As previously reported, chitosan could protect animals from colitis, and help them restore body weight and the overall and histological appearance of the colon. ${ }^{61}$ Likewise, images of HE stained sections revealed that the uninfected and DIC/CS-CPBA
A

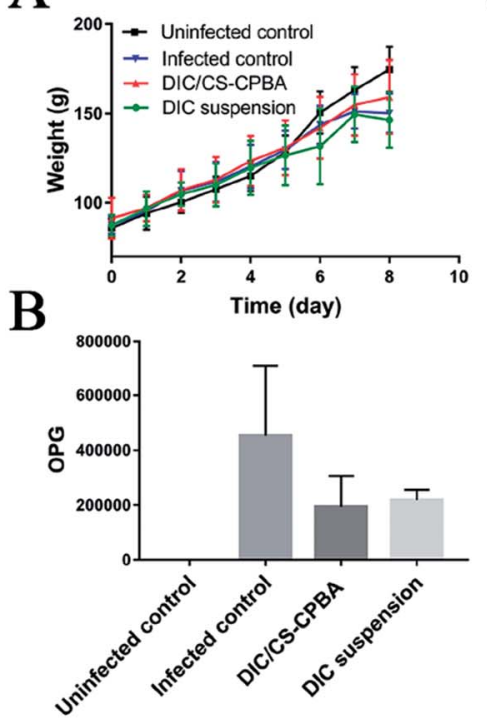

C
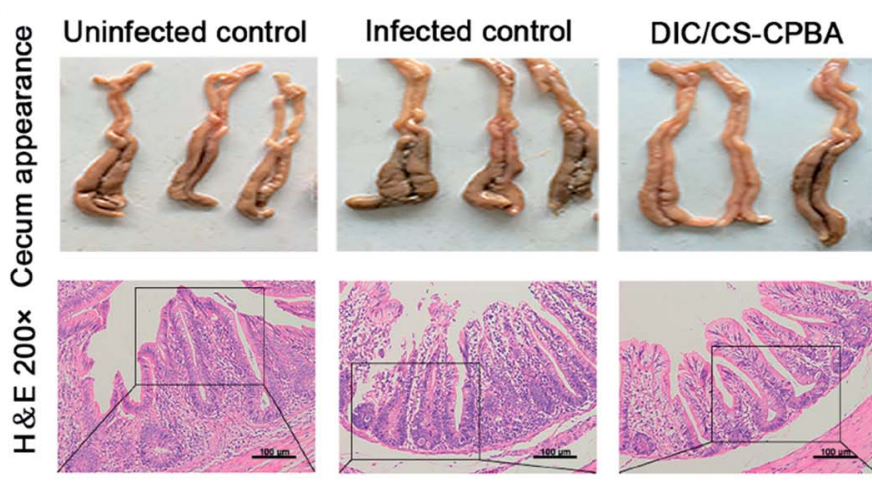

DIC suspension
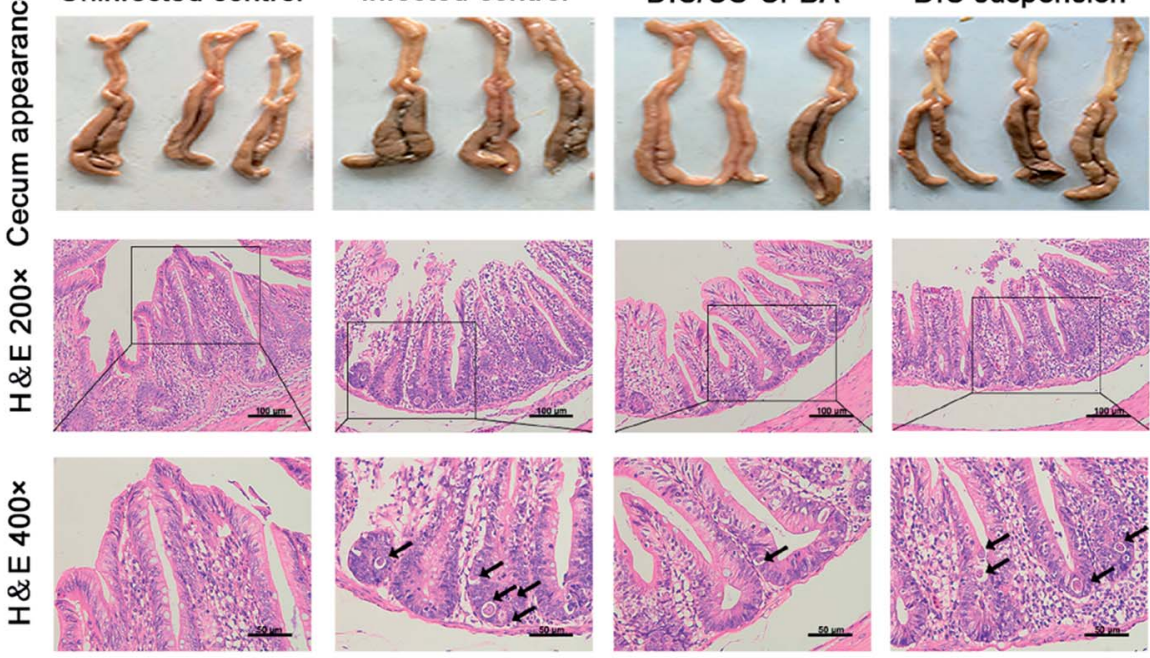

Fig. 6 (A) The average weight change of the chicks administrated by DIC/CS-CPBA micelles and DIC suspensions, and the infected and uninfected groups were set as controls. The number of oocyst per gram of feces (OPG) (B), cecum appearance and H\&E staining images (C) were collected after the experiments. 
micelles treated chicks possessed complete intestinal epithelial villi, and the coccidial oocysts (black arrows) in the epithelial cells were absent or less; while in the other two groups, the intestinal epithelial villi was damaged with more infected coccidia oocysts. The above results demonstrated that DIC/CSCPBA micellar formulation presented a better influence on the protection of the intestinal villi and intestinal epithelial cell prevention from infection caused by the coccidial merozoites, which may lead to a better effect on the treatment of coccidiosis.

\subsection{Pharmacokinetics studies}

For pharmacokinetic studies, the plasma drug concentrationtime curves demonstrated that DIC in CS-CPBA micelles and CMC-Na suspensions shared a similar metabolism process in vivo following oral administration at a single dose of $15 \mathrm{mg} \mathrm{kg}^{-1}$ (Fig. 7). The pharmacokinetic process included two stages was divided into the absorption and distribution process. When the drug directly ingested into the gastrointestinal tract, the rate of drug absorption into the blood circulation was higher than that of the distribution process, and then the drug concentration gradually increased to reach the maximum; at that time the drug absorption rate was less than the distribution rate. It appeared that DIC/CS-CPBA micelles could maintain a higher plasma concentration than that of DIC suspension, suggesting a prolonged circulation of the sufficient concentration of drugs. The pharmacokinetic parameters of DIC were calculated by noncompartmental model via DAS 2.1.1. As can be seen from Table 3, $T_{\max }$ and $\mathrm{AUC}_{0-t}$ values of DIC/CS-CPBA micelles were $8.25 \mathrm{~h}$ and $236.10 \mu \mathrm{g} \mathrm{ml} \mathrm{m}^{-1} \mathrm{~h}^{-1}$, respectively, which were significantly higher than that of drug suspension by approximate 17- and 1.7-folds; while there was no statistically significant difference in the half-life and $C_{\max }$ values. This demonstrated that DIC/CS-CPBA micelles could be used as a long-acting formulation to reduce the number of drug administration and the toxic side effects due to the peak-valley phenomenon of plasma drug concentration.

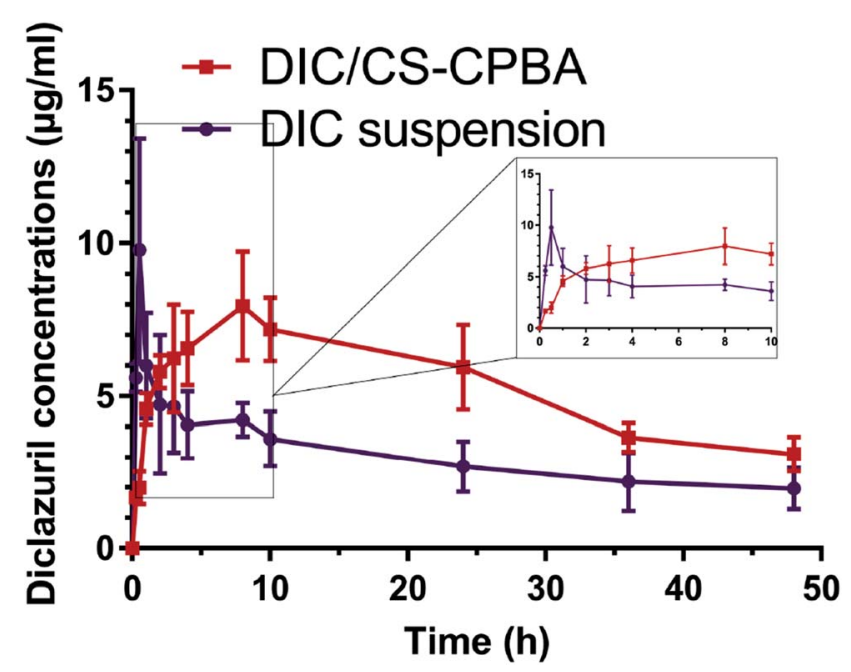

Fig. 7 Mean DIC plasma concentration $\left(\mu \mathrm{g} \mathrm{ml}^{-1}\right.$ ) following a single dose orally administration ( $15 \mathrm{mg} \mathrm{kg}^{-1}$ ) in chickens fed anticoccidial free. Data represents the mean \pm S.D., $n=6$.
Table 3 Pharmacokinetics parameters following a single oral administration (15 mg kg-1 of DIC) to chickens fed anticoccidial free containing ration. Data represents the mean \pm S.D., $n=6^{f}$

\begin{tabular}{llcc}
\hline Parameters & Unit & DIC suspension & DIC/CS-CPBA \\
\hline $\mathrm{AUC}_{0-t}{ }^{a}$ & $\mu \mathrm{g} \mathrm{ml}^{-1} \mathrm{~h}^{-1}$ & $140.40 \pm 7.07$ & $236.10 \pm 28.69^{*}$ \\
$C_{\max }{ }^{c}$ & $\mu \mathrm{g} \mathrm{ml}^{-1}$ & $8.90 \pm 1.20$ & $7.50 \pm 0.63$ \\
$T_{\max }{ }^{d}$ & $\mathrm{~h}$ & $0.48 \pm 0.03$ & $8.25 \pm 0.35^{* *}$ \\
$t_{1 / 2}{ }^{e}$ & $\mathrm{~h}$ & $64.56 \pm 10.55$ & $85.85 \pm 7.59$ \\
$\mathrm{CL}^{e}$ & $\mathrm{ml}(\mathrm{h} \mathrm{g})^{-1}$ & $0.063 \pm 0.0034$ & $0.041 \pm 0.0026$
\end{tabular}

${ }^{a}$ Area under the curve. ${ }^{b}$ Maximum drug concentration. ${ }^{c}$ Time to maximum drug concentration. ${ }^{d}$ Elimination half-life. ${ }^{e}$ Plasma clearance. ${ }^{*} p<0.05$ and ${ }^{* *} p<0.01$ as compared to DIC suspension.

\section{Conclusion}

In this study, chitosan-based polymeric micelles conjugated with CPBA were designed for glucose and $\mathrm{pH}$-triggered sitespecific burst release of anti-coccidia drug. The dual-sensitive DIC-loaded micelles shared spherical shape and nanoscale particle size. DIC/CS-CPBA micelles showed glucose and $\mathrm{pH}$ dual-responsiveness observed by the variations of the micellar morphology and particle size. The cumulative drug release could be accelerated by elevating $\mathrm{pH}$ and glucose concentration. After oral administration, DIC/CS-CPBA micelles possessed much higher anti-coccidia ability, enhanced weight gain efficacy and decreased gastrointestinal damage comparing to the commercially available drug suspension. Pharmacokinetic studies demonstrated that DIC/CS-CPBA micelles gave a higher bioavailability and more stable plasma drug concentration than commercially available drug suspension. Collectively, CPBAmodified chitosan micelles hold a great potential for sitespecific targeted treatment of coccidiosis diseases.

\section{Conflicts of interest}

There are no conflicts to declare.

\section{Acknowledgements}

The authors gratefully acknowledge the financial support from National Key Research and Development Program (2017YFD0501403), National Natural Science Foundation of China (No. 81501582, 81573379 and 81473692) and Natural Science Foundation of Jiangsu Province (No. BK20171390). This study was also supported by National Science and Technology Major Project (No 2017ZX09101001), Development Funds for Priority Academic Programs in Jiangsu Higher Education Institutions and Fostering Plan of University Scientific and Technology Innovation Team of Jiangsu Qing Lan Project (2014).

\section{References}

1 S. E. Macdonald, M. J. Nolan, K. Harman, K. Boulton, D. A. Hume, F. M. Tomley, R. A. Stabler and D. P. Blake, PLoS One, 2017, 12(9), e0184890. 
2 H. W. Peek and W. J. M. Landman, Vet. Q., 2011, 31, 143-161. 3 M. W. Shirley and H. S. Lillehoj, Avian Pathol., 2012, 41, 111121.

4 D. P. Blake and F. M. Tomley, Trends Parasitol., 2014, 30, 1219.

5 T. Li, Y. Li, Y. Xin, Q. Ke, W. Lei, M. N. Mughal, F. Rui, Y. Zhou, S. Bang and J. Zhao, Parasites Vectors, 2017, 10, 137.

6 Z. J. Fang, W. W. Liu, P. Shi, Y. S. Zhang and Z. W. Huang, Avian Biol. Res., 2016, 9, 235-239.

7 B. H. Zhou, L. L. Liu, J. Liu, F. W. Yuan, E. J. Tian and H. W. Wang, Korean J. Parasitol., 2015, 53, 675-682.

8 A. Y. El-Gendi, M. Atef, A. M. Amer and G. M. Kamel, Food Chem. Toxicol., 2010, 48, 3209-3214.

9 C. Wang, C. Han, T. Li, D. Yang, X. Shen, Y. Fan, Y. Xu, W. Zheng, C. Fei and L. Zhang, Vet. Res., 2013, 44, 29.

10 E. J. Tian, B. H. Zhou, X. Y. Wang, J. Zhao, W. Deng and H. W. Wang, Parasitol. Res., 2014, 113, 4057-4064.

11 S. Y. Ma and X. J. Shang, Asian J. Chem., 2012, 24, 5202-5204.

12 A. Bosco, L. Rinaldi, M. E. Morgoglione, M. Santaniello, G. Cappelli, I. Guariglia, L. Nisoli and G. Cringoli, Buffalo Bull., 2013, 32, 1076-1081.

13 D. Hudson, R. Levin and D. Smyth, Absorption from the alimentary tract, 1971.

14 J. Liu, H. Li, X. Jiang, C. Zhang and Q. Ping, Carbohydr. Polym., 2010, 82, 432-439.

15 J. Li, W. Hu, Y. Zhang, H. Tan, X. Yan, L. Zhao and H. Liang, J. Polym. Sci., Part A: Polym. Chem., 2015, 53, 1235-1244.

16 N. A. Hassani, M. Abdouss and S. Faghihi, Mater. Sci. Eng., C, 2014, 41, 91-99.

17 W. Huang, Y. Wang, S. Zhang, L. Huang, D. Hua and X. Zhu, Macromolecules, 2013, 46, 814-818.

18 G. N. Shi, C. N. Zhang, R. Xu, J. F. Niu, H. J. Song, X. Y. Zhang, W. W. Wang, Y. M. Wang, C. Li and X. Q. Wei, Biomaterials, 2017, 113, 191-202.

19 D. Zhao, J. Q. Xu, X. Q. Yi, Q. Zhang, S. X. Cheng, R. X. Zhuo and F. Li, ACS Appl. Mater. Interfaces, 2016, 8, 14845-14854.

20 A. Guerry, S. Cottaz, E. Fleury, J. Bernard and S. Halila, Carbohydr. Polym., 2014, 112, 746-752.

21 J. Wang, M. X. Xu, X. J. Cheng, M. Kong, Y. Liu, C. Feng and X. G. Chen, Carbohydr. Polym., 2016, 136, 867-874.

22 J. Wang and M. Kong, J. Controlled Release, 2017, 259, E94.

23 S. Swiatkiewicz, M. Swiatkiewicz, A. Arczewska-Wlosek and D. Jozefiak, J. Anim. Physiol. Anim. Nutr., 2015, 99, 1-12.

24 H. Kim, Y. J. Kang, S. Kang and K. T. Kim, J. Am. Chem. Soc., 2012, 134, 4030.

25 G. Pan, B. Guo, Y. Ma, W. Cui, F. He, B. Li, H. Yang and K. J. Shea, J. Am. Chem. Soc., 2014, 136, 6203.

26 J. Luo, J. Huang, J. Cong, W. Wei and X. Liu, ACS Appl. Mater. Interfaces, 2017, 9, 7735-7744.

27 W. Lu, Q. Xiong, X. Fei and H. Duan, Biosens. Bioelectron., 2017, 89, 136.

28 M. A. Soriano-Ursúa, E. D. Farfán-García, Y. López-Cabrera, E. Querejeta and J. G. Trujillo-Ferrara, Neurotoxicology, 2014, 40, 8-15.

29 M. R. O'Donovan, C. D. Mee, S. Fenner, A. Teasdale and D. H. Phillips, Mutat. Res., 2011, 724, 1-6.
30 R. J. Ma, H. Yang, Z. Li, G. Liu, X. C. Sun, X. J. Liu, Y. L. An and L. Q. Shi, Biomacromolecules, 2012, 13, 3409-3417.

31 J. Z. Wu, D. H. Bremner, H. Y. Li, S. W. Niu, S. D. Li and L. M. Zhu, Mater. Sci. Eng., C, 2017, 76, 845-855.

32 S. Deshayes, H. Cabral, T. Ishii, Y. Miura, S. Kobayashi, T. Yamashita, A. Matsumoto, Y. Miyahara, N. Nishiyama and K. Kataoka, J. Am. Chem. Soc., 2013, 135, 15501-15507.

33 W. L. A. Brooks and B. S. Sumerlin, Chem. Rev., 2016, 116, 1375-1397.

34 G. Vancoillie and R. Hoogenboom, Sensors, 2016, 16, 1736. 35 Y.-j. Zhang, J.-x. Zheng, J. Li, W.-q. Hu, Y.-y. Sun, H.-z. Liang, L.-l. Zhao and F.-x. Liang, Acta Polym. Sin., 2015, 1020-1027.

36 J. Li, M. Huo, J. Wang, J. Zhou, J. M. Mohammad, Y. Zhang, Q. Zhu, A. Y. Waddad and Q. Zhang, Biomaterials, 2012, 33, 2310-2320.

37 X. B. Fang, J. M. Zhang, X. Xie, D. Liu, C. W. He, J. B. Wan and M. W. Chen, Int. J. Pharm., 2016, 502, 28-37.

38 G. B. Shrikhande and A. K. Satpute, Vet. World, 2008, 1, 207. 39 J. Johnson and W. M. Reid, Exp. Parasitol., 1970, 28, 30.

40 A. J. Tanweer, N. Chand, U. Saddique, C. A. Bailey and R. U. Khan, Parasitol. Res., 2014, 113, 2951-2960.

41 D. Zhang, G. Yu, Z. Long, G. Yang and B. Wang, Carbohydr. Polym., 2016, 140, 228-232.

42 D. Shi, M. Ran, H. Huang, L. Zhang, X. Li, M. Chen and M. Akashi, Polym. Chem., 2016, 7, 6779-6788.

43 D. Abdelhamid, H. Arslan, Y. Zhang and K. E. Uhrich, Polym. Chem., 2014, 5, 1457.

44 C. Liu, Y. Guan, Y. Su, L. Zhao, F. Meng, Y. Yao and J. Luo, RSC Adv., 2017, 7, 11021-11029.

45 Y. Zhang, Q. Li, W. J. Welsh, P. V. Moghe and K. E. Uhrich, Biomaterials, 2016, 84, 230-240.

46 X. Zhu, A. Tsend-Ayush, Z. Yuan, J. Wen, J. Cai, S. Luo, J. Yao, J. Bian, L. Yin, J. Zhou and J. Yao, Int. J. Pharm., 2017, 529, 451-464.

47 R. A. M. Osmani, S. Thirumaleshwar, R. R. Bhosale and P. K. Kulkarni, Der Pharmacia Sinica, 2014, 5, 7-21.

48 S. H. Chang, H. T. Lin, G. J. Wu and G. J. Tsai, Carbohydr. Polym., 2015, 134, 74-81.

49 J. R. Pappenheimer, Am. J. Physiol., 1993, 265, 409-417.

50 G. L. Kellett, J. Physiol., 2001, 531, 585-595.

51 Z. Ke, Z. Zhang, H. Wu, X. Jia and Y. Wang, Int. J. Pharm., 2017, 518, 193-202.

52 M. Rajan, P. Krishnan, P. Pradeepkumar, M. Jeyanthinath, M. Jeyaraj, M. P. Ling, P. Arulselvan, A. Higuchi, M. A. Munusamy, R. Arumugam, G. Benelli, K. Murugan and S. S. Kumar, RSC Adv., 2017, 7, 46271-46285.

53 M. Huo, A. Zou, C. Yao, Y. Zhang, J. Zhou, J. Wang, Q. Zhu, J. Li and Q. Zhang, Biomaterials, 2012, 33, 6393-6407.

54 Z. Wu, S. Zhang, X. Zhang, S. Shu, T. Chu and D. Yu, J. Pharm. Sci., 2011, 100, 2278-2286.

55 V. Tsiouris, I. Georgopoulou, C. Batzios, N. Pappaioannou, A. Diakou, E. Petridou, R. Ducatelle and P. Fortomaris, Avian Pathol., 2013, 42, 163-170.

56 C. G. Olnood, S. S. M. Beski, P. A. Iji and M. Choct, Animal Nutrition, 2015, 1, 192-202.

57 J. L. Dorne, M. L. Fernándezcruz, U. Bertelsen, D. W. Renshaw, K. Peltonen, A. Anadon, A. Feil, 
P. Sanders, P. Wester and J. Finkgremmels, Toxicol. Appl. Pharmacol., 2013, 270, 196.

58 EFSA (European Food Safety Authority), EFSA J., 2007, 506, 132.

59 B. Krajewska, P. Wydro and A. Jańczyk, Biomacromolecules, 2011, 12, 4144-4152.
60 M. C. Cruz-Romero, T. Murphy, M. Morris, E. Cummins and J. P. Kerry, Food Control, 2013, 34, 393-397.

61 H. Hyun, S. Hashimoto-Hill, M. Kim, M. D. Tsifansky, C. H. Kim and Y. Yeo, ACS Biomater. Sci. Eng., 2017, 3, 1853-1860. 